\title{
ON RIEMANN-ROCH FORMULAS FOR MULTIPLICITIES
}

\author{
ECKHARD MEINRENKEN
}

\section{INTRODUCTION}

Let $(M, \omega)$ be a compact Kähler manifold, and let $\tau: L \rightarrow M$ be a holomorphic line bundle over $M$ with Hermitian fiber metric $h .(L, h)$ is called quantizing if $-2 \pi i \omega$ is the curvature of the canonical Hermitian connection on $L$. Let $H^{i}(M, \mathcal{O}(L))$ be the $i$ th cohomology group for the sheaf of germs of holomorphic sections. By the Riemann-Roch formula of Hirzebruch and Atiyah-Singer [4], the Euler number

$$
\operatorname{Eul}(L):=\sum_{i}(-1)^{i} \operatorname{dim} H^{i}(M, \mathcal{O}(L))
$$

is equal to the characteristic number

$$
\operatorname{Eul}(L)=\int_{M} \operatorname{Td}(M) C h(L),
$$

where $T d(M)$ is the Todd class and $C h(L)=e^{[\omega]}$ the Chern character. Recall that if $L$ is "sufficiently positive", in particular if one replaces $L$ by some sufficiently high tensor power, all the cohomology groups with $i>0$ are zero by Kodaira's vanishing theorem, so in this case (2) gives a formula for the dimension of the space $H^{0}(M, \mathcal{O}(L))=\Gamma_{h o l}(M, L)$ of holomorphic sections.

Let $G$ be a compact, connected Lie group that acts on $M$ by Kähler diffeomorphisms $\Phi: G \times M \rightarrow M$, with an equivariant moment map $J: M \rightarrow \mathfrak{g}^{*}$. Suppose also that $\Phi$ lifts to Hermitian bundle automorphisms of $L \rightarrow M$, according to the rules of geometric quantization. The corresponding virtual representation of $G$ on $\sum(-1)^{i} H^{i}(M, \mathcal{O}(L))$ may then be regarded as the "quantization" of the classical action $\Phi$. Its character $\chi$ is the element of the representation $\operatorname{ring} R(G)$ defined by

$$
\chi(g):=\sum_{i}(-1)^{i} \operatorname{tr}\left(g \mid H^{i}(M, \mathcal{O}(L))\right) .
$$

From the equivariant Riemann-Roch formula of Atiyah-Segal-Singer [3, 4], one has an expression for $\chi(g)$ as the evaluation of certain characteristic classes on the fixed point set $M^{g}=\{x \in M \mid g \cdot x=x\}$ (which is a Kähler submanifold of $M$ ): Let $C h^{g}\left(L \mid M^{g}\right)=c_{L}(g)^{-1} C h\left(L \mid M^{g}\right)$, where $c_{L}(g) \in S^{1}$ is the (locally constant) action of $g$ on $L \mid M^{g}$. Denote by $N^{g}$ the normal bundle of $M^{g}$ in $M$, by $F\left(N^{g}\right)$ its curvature, and let

$$
D^{g}\left(N^{g}\right)=\operatorname{det}\left(I-\left(g^{\sharp}\right) e^{-\frac{i}{2 \pi} F\left(N^{g}\right)}\right)
$$

Received by the editors June 16, 1994

1991 Mathematics Subject Classification. Primary 53C15, 58F05, 58G07. 
where $g^{\sharp}$ is the automorphism of $N^{g}$ defined by $g$. Then

$$
\chi(g)=\int_{M^{g}} \frac{T d\left(M^{g}\right) C h^{g}\left(L \mid M^{g}\right)}{D^{g}\left(N^{g}\right)} .
$$

By a theorem of Guillemin and Sternberg [16], there are also Riemann-Roch formulas for the multiplicities of the irreducible components of the above representation, at least if certain regularity assumptions are satisfied. Let $T \subset G$ be a maximal torus, and $\mathfrak{g}=\mathfrak{t} \oplus[\mathfrak{t}, \mathfrak{g}]$ the corresponding decomposition of the Lie algebra. Choose a fundamental Weyl chamber $\mathfrak{t}_{+}^{*} \subset \mathfrak{t}^{*} \subset \mathfrak{g}^{*}$, let $\Lambda^{*} \subset \mathfrak{t}^{*}$ be the integral lattice, and $\Lambda_{+}^{*}=\Lambda \cap \mathfrak{t}_{+}^{*}$ the dominant weights. For a given lattice point $\mu \in \Lambda_{+}^{*}$, let $V_{\mu}$ denote the corresponding irreducible representation with highest weight $\mu$, and define the multiplicity $N(\mu)$ by the alternating sum

$$
N(\mu):=\sum_{i}(-1)^{i} \operatorname{dim}\left(V_{\mu}^{*} \otimes H^{i}(M, \mathcal{O}(L))\right)^{G} .
$$

Suppose that $\mu \in \Lambda_{+}^{*}$ is a regular value of $J$, or equivalently that the action of the isotropy group $G_{\mu}$ on $J^{-1}(\mu)$ is locally free. If the action is in fact free, the reduced space $M_{\mu}=J^{-1}(\mu) / G_{\mu}$ is a smooth symplectic manifold, and it is well-known that it acquires a natural Kähler structure, together with a quantizing line bundle $L_{\mu}$. The main result of [16] is that the multiplicity of $\mu$ in $\Gamma_{h o l}(M, L)$ is equal to the dimension of the space $\Gamma_{h o l}\left(M_{\mu}, L_{\mu}\right)$, so in particular $N(\mu)$ is given by the Euler number of $L_{\mu}$ if $L$ is sufficiently positive:

Theorem 1.1 (V. Guillemin, S. Sternberg [16]). If the action of $G_{\mu}$ on $J^{-1}(\mu)$ is free, and if $L$ is sufficiently positive,

$$
N(\mu)=\int_{M_{\mu}} \operatorname{Td}\left(M_{\mu}\right) C h\left(L_{\mu}\right) .
$$

The "physical" interpretation of this theorem is that reduction and quantization commute.

In practice, one is often dealing with situations where the action is only locally free. In this case, the reduced space is in general just an orbifold (or V-manifold) in the sense of Satake [21], which means (roughly) that it is locally the quotient of a manifold by a finite group. Moreover, the reduced line bundle is in general just an orbifold-bundle, that is, at some points the fiber of $L_{\mu}$ is not $\mathbb{C}$, but its quotient by a finite group. Guillemin and Sternberg conjectured that in this case, the right hand side of (7) has to be replaced by the expression from T. Kawasaki's Riemann-Roch formula for orbifolds [20]. It was proved by R. Sjamaar [22] that this assertion is true if $L$ is sufficiently positive, and if $L_{\mu}$ is an honest line bundle. In fact, his approach also covers the truly singular case where $\mu$ is not even a regular value, by using Kirwan's partial desingularization procedure to reduce it to the orbifold case. On the other hand, the condition that $L_{\mu}$ be a genuine line bundle is rather restrictive.

Moreover, the proofs of Guillemin-Sternberg and Sjamaar rely heavily on complex geometry arguments, while Theorem 1.1 also makes sense for the non-Kähler case. In fact, for every quantizable symplectic manifold $M$, one can define $\chi(g)$ as the equivariant index of the $\operatorname{Spin}^{c}$-Dirac operator $\not$ associated to $L \rightarrow M$. The construction of $\not$ involves some choices, for instance the choice of a compatible positive almost complex structure on $M$, but the index does not depend on that, and is again given by (5). 
It is the aim of the present paper to give a different proof of the GuilleminSternberg conjecture (for $\mu$ a regular value), which covers both the orbifold- and the non-Kähler case.

The method we use is motivated by recent work of V. Guillemin [13], who used localization techniques from equivariant cohomology to establish the connection between the multiplicity formula (7) and a certain formula for counting lattice points in polytopes. This formula is known to be true in various interesting cases, and for these gives a new proof of (7) without using complex geometry techniques.

We will also use equivariant cohomology, but in a slightly different guise. The main idea is to consider the rescaled problem, where we replace

$$
\omega \leadsto m \omega, L \leadsto L^{m}, J \leadsto m J, \mu \leadsto m \mu
$$

for $m \in \mathbb{N}$. Let $N^{(m)}: \Lambda_{+}^{*} \rightarrow \mathbb{Z}$ be the corresponding multiplicity function.

Our starting point will be the equivariant Riemann-Roch formula, but in a form due to Berline and Vergne [6], involving equivariant characteristic classes. By a stationary phase version of the localization formula of Jeffrey-Kirwan-Witten [19, 25], we pass from equivariant characteristic classes to (ordinary) characteristic classes on the reduced spaces. This leads to the desired multiplicity formula for $N^{(m)}(m \mu)$, up to an error term $O\left(m^{-\infty}\right)$.

Since the multiplicities are integers, one easily finds that the error term is zero for large $m$. To investigate the general dependence of $N^{(m)}(m \mu)$ on $m$, we use a different expression for $N^{(m)}(m \mu)$ via the number of lattice points in certain polytopes. If $J(M)$ is contained in the set of regular elements of $\mathfrak{g}^{*}$, in particular in the abelian case, this analysis turns out to be sufficiently good to show that the above error term is zero for all $m$.

\section{ACKNOWLEDGEMENTS}

I would like to thank V. Guillemin, J. Kalkman, E. Lerman, R. Sjamaar and C. Woodward for useful comments and discussions. After completing this article, I learned that M. Vergne has found a different proof of the Guillemin-Sternberg conjecture for the abelian case, see [23].

\section{Statement of the Result}

In order to state the result, we have to give a more precise description of the reduced space and its singular strata. Suppose that $\mu \in \Lambda_{+}^{*}$ is a regular value of $J$. Recall first the shifting-trick to express $M_{\mu}$ as a reduced space at the zero level set: Let $O=G$. $\mu$ be the coadjoint orbit through $\mu$, equipped with its usual Kirillov Kähler structure, and let $O^{-}$denote $O$ with the opposite Kähler structure. The action of $G$ on $O$ is Hamiltonian, with moment map $\Psi$ the embedding into $\mathfrak{g}^{*}$. Then $\tilde{M}=M \times O^{-}$is a Kähler manifold, and the diagonal action of $G$ is Hamiltonian, with moment map $\tilde{J}=J-\Psi$. There are canonical identifications

$$
M_{\mu}=J^{-1}(\mu) / G_{\mu} \cong J^{-1}(O) / G \cong \tilde{J}^{-1}(0) / G
$$

By Kostant's version of the Borel-Weil-Bott theorem, one also has a natural quantizing bundle $\Xi \rightarrow O$, and the irreducible representation $V_{\mu}$ corresponding to $\mu$ gets realized as the space of holomorphic sections of $\Xi$. (The higher order cohomology groups $H^{i}(O, \mathcal{O}(\Xi))$ vanish.) The tensor product $\tilde{L}:=L \otimes \Xi^{*}$ quantizes $\tilde{M}$, and 
there is an isomorphism

$$
H^{i}(\tilde{M}, \mathcal{O}(\tilde{L})) \cong V_{\mu}^{*} \otimes H^{i}(M, \mathcal{O}(L))
$$

Hence $N(\mu)=\operatorname{Eul}(\tilde{L})$, which is the quantum counterpart of the shifting-trick.

Using the shifting-trick, it is enough to consider the case $\mu=0$. The reduced space $M_{0}$ inherits a natural Kähler structure from $M$ (see [16]), and the reduced bundle $L_{0}=\left(L \mid J^{-1}(0)\right) / G$ yields a quantizing orbifold-line bundle. Note however that $L_{0}$ need not be an honest line bundle, not even over the smooth part of $M_{0}$. Sections of an orbifold bundle are defined as coming from invariant sections for the local orbifold charts, so all sections of $L_{0}$ have to vanish at points were the fiber is not $\mathbb{C}$.

Let us regard $P=J^{-1}(0)$ as an orbifold-principal bundle over $M_{0}=J^{-1}(0) / G$. Following [20, 12], we introduce

$$
\tilde{P}=\{(x, g) \mid x \in P, g . x=x\} \subset P \times G,
$$

and its quotient $\Sigma=\tilde{P} / G$ under the locally free action $h .(x, g)=\left(h . x, h g h^{-1}\right)$. The projection of $\tilde{P}$ to the second factor descends to a locally constant mapping

$$
\tau: \Sigma \rightarrow \operatorname{Conj}(G)
$$

to the set of conjugacy classes. For $g \in G$, let $(g)=\operatorname{Ad}(G) \cdot g$ denote the corresponding conjugacy class, and $\Sigma_{g}$ its preimage under $\tau$. There is a natural identification $\Sigma_{g}=P^{g} / Z_{g}$, where $P^{g}=M^{g} \cap P$ is the fixed point manifold and $Z_{g}$ the centralizer of $g$ in $G$. Since the fixed point set $M^{g} \subset M$ is a Kähler submanifold, and the action of $Z_{g}$ on $M^{g}$ is Hamiltonian with the restriction of $J$ serving as a moment map, this makes it clear that $\Sigma$ is a Kähler orbifold (with several components of different dimensions). Note that this Kähler structure does not depend on the choice of the representative for $(g)$. Observe also that $\Sigma_{e} \cong M_{0}$.

The collection of bundles $\left(L \mid P^{g}\right) / Z_{g}$ defines a quantizing orbifold line bundle $L_{\Sigma} \rightarrow \Sigma$. As above, let $c_{L}(g) \in S^{1}$ be the locally constant action of $g$ on $L \mid P^{g}$, denote by $c_{\Sigma}: \Sigma \rightarrow S^{1}$ the function defined by the $c_{L}(g)$ 's, and let $C h^{\Sigma}\left(L_{\Sigma}\right)$ be the cohomology class defined by

$$
C h^{\Sigma}\left(L_{\Sigma}\right)=c_{\Sigma}^{-1} e^{\omega_{\Sigma}}
$$

where $\omega_{\Sigma}$ is the Kähler form on $\Sigma$.

Consider now the natural mapping $f: \Sigma \rightarrow M_{0}$, sending $G .(x, g) \rightarrow G$. $x$. In a local orbifold chart, the tangent space to $\Sigma$ at $G$. $(x, g)$ is isomorphic to

$$
T_{x}\left(M^{g} \cap J^{-1}(0)\right) / T_{x}\left(Z_{g} \cdot x\right),
$$

while the tangent space to $M_{0}$ at $G . x$ is $T_{x}\left(J^{-1}(0)\right) / T_{x}(G . x)$. From this, it is easy to see that $f$ is a Kähler immersion. Let $N_{\Sigma} \rightarrow \Sigma$ be the normal bundle of this immersion, and denote by $g^{\sharp}$ the automorphism of $N_{\Sigma} \mid \Sigma_{g}$ induced by the action of $g$. Then the collection of differential forms

$$
\operatorname{det}\left(I-\left(g^{\sharp}\right) e^{-\frac{i}{2 \pi} F\left(N_{\Sigma}\right)}\right),
$$

where $F\left(N_{\Sigma}\right)$ is the curvature of $N_{\Sigma}$, defines a characteristic class $D^{\Sigma}\left(N_{\Sigma}\right)$.

Finally, for each connected component $\Sigma_{i}$ of $\Sigma$, let $d_{i}$ be the number of elements in a generic stabilizer for the $G$-action on the corresponding component $\tilde{P}_{i}$, and $d_{\Sigma}: \Sigma \rightarrow \mathbb{N}$ the function defined by the $d_{i}$ 's. 
For general values $\mu \in \Lambda_{+}^{*}$, let $\Sigma_{\mu}, L_{\mu}$ etc. be defined by means of the shiftingtrick. The main result of this paper is the following.

Theorem 2.1 (Multiplicity Formula). If $\mu \in \Lambda_{+}^{*}$ is a regular value of $J$, the multiplicities $N^{(m)}(m \mu)$ are for $m>>0$ given by the formula

$$
N^{(m)}(m \mu)=\int_{\Sigma_{\mu}} \frac{1}{d_{\Sigma_{\mu}}} \frac{\operatorname{Td}\left(\Sigma_{\mu}\right) C h^{\Sigma_{\mu}}\left(L_{\Sigma_{\mu}}^{m}\right)}{D^{\Sigma_{\mu}}\left(N_{\Sigma_{\mu}}\right)} .
$$

If the image of the moment map, $J(M)$, is contained in $\mathfrak{g}_{r e g}^{*}$ (the set of regular elements of $\left.\mathfrak{g}^{*}\right)$, one may set $m=1$ in this formula:

$$
N(\mu)=\int_{\Sigma_{\mu}} \frac{1}{d_{\Sigma_{\mu}}} \frac{T d\left(\Sigma_{\mu}\right) C h^{\Sigma_{\mu}}\left(L_{\Sigma_{\mu}}\right)}{D^{\Sigma_{\mu}}\left(N_{\Sigma_{\mu}}\right)},
$$

In particular, this is the case if $G$ is abelian.

Remarks. (1) Comparing the right hand side of (16) to Kawasaki's Riemann-Roch formula for orbifolds [20], the theorem says that $N(\mu)$ is equal to the Euler number of the orbifold-bundle $L_{\mu} \rightarrow M_{\mu}$. In particular, $N(\mu)$ is zero if the fiber of $L_{\mu}$ over the smooth stratum of $M_{\mu}$ is a nontrivial quotient of $\mathbb{C}$.

(2) Let $\Delta=J(M) \cap \mathfrak{t}_{+}^{*}$, which is a convex polytope by a result of GuilleminSternberg and Kirwan, and $\Delta^{*} \subset \Delta$ the set of regular values. By the DuistermaatHeckman theorem [9], the diffeotype of the reduced space $M_{\mu}$ (and of course also of $\left.\Sigma_{\mu}\right)$ does not change as $\mu$ varies in a connected component of $\operatorname{int}\left(\mathfrak{t}_{+}^{*}\right) \cap \Delta^{*}$, and the cohomology class of the symplectic form $\omega_{\mu}$ varies linearly. In particular, the symplectic volume $\operatorname{Vol}\left(M_{\mu}\right)$ is a polynomial on these connected components. If the action of $G_{\mu}$ on $J^{-1}(\mu)$ is free, so that $\Sigma_{\mu}=M_{\mu}$, the right hand side in (16) is equal to a polynomial as well, since all that varies is the Chern character $C h\left(L_{\mu}\right)=e^{\omega_{\mu}}$. In the orbifold case, the behaviour is slightly more complicated: For $\mu \in \Lambda_{+}^{*}$ in any given connected component of $\operatorname{int}\left(\Delta^{*}\right)$, and any connected component $\Sigma_{\mu, j}$ of $\Sigma_{\mu}$,

$$
C h^{\Sigma_{\mu}}\left(L_{\Sigma_{\mu}}\right) \mid \Sigma_{\mu, j}=\rho_{\mu}\left(g_{j}\right) c_{L}\left(g_{j}^{-1}\right) e^{\omega_{\Sigma_{\mu}}},
$$

where $g_{j} \in G_{\mu}$ represents $\tau\left(\Sigma_{\mu, j}\right)$, and $\rho_{\mu}: G_{\mu} \rightarrow S^{1}$ is defined by $\rho_{\mu}(\exp (\xi))=$ $e^{2 \pi i\langle\mu, \xi\rangle}$. Hence, the right hand side of (16) is of the form

$$
N(\mu)=\sum_{j} \rho_{\mu}\left(g_{j}\right) c_{L}\left(g_{j}^{-1}\right) p_{j}(\mu)
$$

where the $p_{j}$ are polynomials of degree $\frac{1}{2} \operatorname{dim}\left(\Sigma_{\mu, j}\right)$.

(3) Since $C h\left(\left(L^{m}\right)_{m \mu}\right)=e^{m \omega_{\mu}}$, the right hand side of (15) is a polynomial in $m$ if the $G_{\mu}$-action on $J^{-1}(\mu)$ is free. In the orbifold-case, this is not true in general since

$$
C h^{\Sigma_{\mu}}\left(L_{\Sigma_{\mu}}^{m}\right) \mid \Sigma_{\mu, j}=\rho_{\mu}\left(g_{j}\right)^{m} c_{L}\left(g_{j}^{-1}\right)^{m} e^{m \omega_{\Sigma_{\mu}}} .
$$

Definition 2.1 (Ehrhart [11]). A function $f: \mathbb{N} \rightarrow \mathbb{C}$ is called an arithmetic polynomial, if for some $k \in \mathbb{N}$, all the functions

$$
q_{j}(m)=f(k m-j), j=0, \ldots, k-1,
$$

are polynomials. $k$ is called the period of $f$. 
Equivalently, $f$ is an arithmetic polynomial if and only if it can be written in the form

$$
f(m)=\sum_{l=0}^{k-1} \exp \left(2 \pi i \frac{l m}{k}\right) p_{l}(m),
$$

where the $p_{l}$ are polynomials. Taking $k$ such that $g^{k}=e$ for all $(g) \in \tau\left(\Sigma_{\mu}\right)$, the right hand side of (15) clearly has this property.

(4) Our proof of Theorem 2.1 does not really use the assumption that $M$ is Kähler. Everything will be derived from the equivariant Riemann-Roch formula (3), which is of course valid in much more general situations. Suppose for instance that $M$ is an arbitrary compact symplectic manifold, equipped with a Hamiltonian $G$-action, and that these data are quantizable. Then one can always choose a compatible, invariant almost Kähler structure, and replace the virtual space $\sum(-1)^{i} H^{i}(M, \mathcal{O}(L))$ with the index space of the Spin ${ }^{c}$-Dirac operator associated to $L \rightarrow M$ (see $[7,13])$. As an immediate consequence of the Berline-Vergne formula for the character, Theorem 3.1 below, the multiplicities $N(\mu)$ defined in this way do not depend on the choice of the almost Kähler structure or of the quantizing line bundle $L$.

(5) Suppose that $M$ is an almost complex manifold, $L \rightarrow M$ an Hermitian line bundle with connection, and $\omega$ defined by $\omega=\frac{i}{2 \pi} F(L)$. Suppose $G$ acts on $L \rightarrow M$, preserving the connection, and let $J$ be the corresponding moment map. Even if $\omega$ is degenerate, it is still true that the action of $G_{\mu}$ on $J^{-1}(\mu)$ is locally free if $\mu$ is a regular value of $J$, and one can form a reduced space $\left(M_{\mu}, \omega_{\mu}\right)$. In general, there is no natural 'reduced' almost complex structure on $M_{\mu}$, but our proof will show that Theorem 2.1 is true for this setting, provided one replaces $\operatorname{Td}\left(M_{\mu}\right)$ by $\kappa_{\mu}\left(T d_{\mathfrak{g}}(M) j_{\mathfrak{g}}\right)$ (the notation will be explained below).

Example . Let $M=\mathbb{C} P(2)$, equipped with the Fubini-Study Kähler form $\omega_{F S}$. Let $G=S^{1}$ act by

$$
e^{i \phi} \cdot\left[z_{0}: z_{1}: z_{2}\right]=\left[e^{i \phi} z_{0}: e^{-i \phi} z_{1}: z_{2}\right] .
$$

This action is Hamiltonian, and has a moment map

$$
J\left(\left[z_{0}: z_{1}: z_{2}\right]\right)=\frac{\left|z_{1}\right|^{2}-\left|z_{0}\right|^{2}}{\left|z_{0}\right|^{2}+\left|z_{1}\right|^{2}+\left|z_{2}\right|^{2}} .
$$

The dual of the tautological line bundle serves as a quantizing line bundle $L$. We also consider the tensor powers $L^{m}$, which are quantizing line bundles for $M^{(m)}=$ $\mathbb{C} P(2)$, with symplectic form $\omega^{(m)}=m \omega_{F S}$. By Kodaira's vanishing theorem, $H^{i}\left(M, \mathcal{O}\left(L^{m}\right)\right)=0$ for all $i>0, m \in \mathbb{N}$. If we identify the spaces $\Gamma_{h o l}\left(M, L^{m}\right)$ with the homogeneous polynomials of degree $m$ on $\mathbb{C}^{3}$, the representation of $S^{1}$ is induced by the action $e^{i \phi} \cdot\left(z_{0}, z_{1}, z_{2}\right)=\left(e^{i \phi} z_{0}, e^{-i \phi} z_{1}, z_{2}\right)$ on $\mathbb{C}^{3}$. The isotypical subspace of $\Gamma_{h o l}\left(M, L^{m}\right)$ corresponding to the weight $l \in \mathbb{Z}$ is, for $l \geq 0$, spanned by

$$
z_{0}^{l} z_{2}^{m-l}, z_{0}^{l+1} z_{1} z_{2}^{m-l-2}, \ldots, z_{0}^{l+r} z_{1}^{r} z_{2}^{m-l-2 r}
$$

with $r=\left[\frac{m-l}{2}\right]$. For $l \leq 0$, the roles of $z_{0}$ and $z_{1}$ are reversed. Thus

$$
N^{(m)}(l)=\left\{\begin{array}{ll}
1+\left[\frac{m-|l|}{2}\right] & \text { if }|l| \leq m \\
0 & \text { otherwise }
\end{array},\right.
$$

for all $l \in \mathbb{Z}, m \in \mathbb{N}$. 
On the other hand, the image of the moment map $J^{(m)}=m J$ is the interval $-m \leq \mu \leq m$, with critical values at $-m, 0, m$. If $0<|l|<m$, the level set $(m J)^{-1}(l)$ consists of two orbit type strata: On the set where $z_{2} \neq 0$, the action is free, and on the set where $z_{2}=0$, the stabilizer is $\mathbb{Z}_{2}=\{e, g\}$. Hence, the reduced space $M_{\mu}^{(m)}$ is an orbifold with a $\mathbb{Z}_{2}$ singularity (the "teardrop-orbifold"), and $\Sigma_{\mu}^{(m)}$ consists of two connected components: a copy of $M_{\mu}^{(m)}$ and a copy of the singular point.

Using the Duistermaat-Heckman theorem [9], it is easy to compute the symplectic volume of $M_{\mu}^{(m)}$, with result $\int \omega_{\mu}^{(m)}=(m-|\mu|) / 2$ for $|\mu| \leq m$. For the Todd number of the $\mathbb{Z}_{2}$ teardrop orbifold one has $\int T d\left(M_{\mu}^{(m)}\right)=3 / 4$ (this can be seen for example by writing down Kawasaki's Riemann-Roch formula for the trivial bundle). Therefore, the contribution of $M_{\mu}^{(m)}$ to (16) becomes

$$
p_{e}^{(m)}(\mu)=\int_{M_{\mu}^{(m)}} T d\left(M_{\mu}^{(m)}\right) e^{\omega_{\mu}^{(m)}}=3 / 4+(m-|\mu|) / 2 .
$$

For the singular point, we have the multiplicity $d_{g}=2$, and the action of $g$ on the normal bundle $N_{\Sigma}$ is multiplication by $(-1)$. Hence

$$
p_{g}^{(m)}(\mu)=\frac{1}{2}(1-(-1))^{-1}=\frac{1}{4} .
$$

Since $c_{L^{m}}\left(g^{-1}\right)=(-1)^{m}$ and $\rho_{l}(g)=(-1)^{l}$, this gives

$$
N^{(m)}(l)=3 / 4+(m-|l|) / 2+(-1)^{m-l} / 4=1+[(m-|l|) / 2]
$$

for $|l| \leq m$, in agreement with the above calculation.

Note that for $m-|l|$ even, the fiber of $L_{l}^{m}$ at the singular point is $\mathbb{C}$, whereas for $m-|l|$ odd it is $\mathbb{C} / \mathbb{Z}_{2}$. This means that for $m-|l|$ odd, all holomorphic sections of $L_{l}^{m}$ have to vanish at the singular point. Again, this fits with the above explicit formulas.

\section{Some Equivariant COHOMOLOGY}

We start by reviewing Cartan's model for equivariant cohomology, following Berline and Vergne [6]. Let $M$ be a compact manifold, $G$ a compact Lie group, and $\Phi: G \times M \rightarrow M$ a smooth action. Denote by $\mathcal{A}_{G}(M)$ the space of $G$-invariant polynomial mappings $\sigma: \mathfrak{g} \rightarrow \mathcal{A}(M)$, that is, $\sigma(\xi)$ depends polynomially on $\xi$ and satisfies the equivariance property

$$
\sigma\left(\operatorname{Ad}_{g}(\xi)\right)=\Phi_{g^{-1}}^{*}(\sigma(\xi))
$$

The elements of $\mathcal{A}_{G}(M)$ are called equivariant differential forms, and the space $\mathcal{A}_{G}(M)$ is preserved under the equivariant differential

$$
\mathrm{d}_{\mathfrak{g}}: \mathcal{A}_{G}(M) \rightarrow \mathcal{A}_{G}(M),\left(\mathrm{d}_{\mathfrak{g}} \sigma\right)(\xi)=\mathrm{d}(\sigma(\xi))+2 \pi i\left(\iota\left(\xi_{M}\right) \sigma(\xi)\right) .
$$

Here, $\xi_{M}$ denotes the fundamental vector field, i.e. the generating vector field of the flow $(t, p) \mapsto \exp (t \xi) \cdot p$.

Equivariance together with Cartan's identity for the Lie derivative, $\mathcal{L}_{Y}=\iota_{Y} \circ$ $\mathrm{d}+\mathrm{d} \circ \iota_{Y}$, implies $\mathrm{d}_{\mathfrak{g}}^{2}=0$. The cohomology $H_{G}(M)$ of the complex $\left(\mathcal{A}_{G}(M), \mathrm{d}_{\mathfrak{g}}\right)$ is called the equivariant cohomology. One can show that if the action of $G$ on $M$ is locally free, the pullback mapping $\mathcal{A}(M / G) \rightarrow \mathcal{A}(M)_{\text {hor }}^{G} \hookrightarrow \mathcal{A}_{G}(M)$ gives rise 
to an isomorphism $H(M / G) \rightarrow H_{G}(M)$. After choosing a principal connection on $M \rightarrow M / G$, the inverse is induced on the level of forms by the mapping

$$
\mathcal{A}_{G}(M) \rightarrow \mathcal{A}(M)^{G} \rightarrow \mathcal{A}(M)_{h o r}^{G} \cong \mathcal{A}(M / G)
$$

given by substituting $\frac{i}{2 \pi}$ times the curvature in the $\mathfrak{g}$-slot, followed by projection onto the horizontal part (for a proof, see [10]).

For what follows, it will be necessary to relax the polynomial dependence on $\xi$ to analytic dependence, possibly defined only on some neighborhood of $0 \in \mathfrak{g}$. We will denote the corresponding space of equivariant forms by $\mathcal{A}_{G}^{\omega}(M)$, and the cohomology by $H_{G}^{\omega}(M)$.

Suppose now that $\mathcal{V} \rightarrow M$ is a $G$-equivariant Hermitian vector bundle over $M$, with fiber dimension $N$. Let $\mathcal{A}(M, \mathcal{V})$ be the bundle-valued differential forms, and $\mathcal{A}_{G}(M, \mathcal{V})$ their equivariant counterpart. For each $G$-invariant Hermitian connection $\nabla: \mathcal{A}(M, \mathcal{V}) \rightarrow \mathcal{A}(M, \mathcal{V})$, the moment map $\mu \in \mathcal{A}_{G}(M, \operatorname{End}(\mathcal{V}))$ of Berline and Vergne is defined by

$$
\mu(\xi) . \sigma:=\xi . \sigma-\nabla_{\xi_{M}} \sigma,
$$

where $\sigma \rightarrow \xi . \sigma$ denotes the representation of $\mathfrak{g}$ on the space of sections. Geometrically, $\mu(\xi)$ is the vertical part (with respect to the connection) of the fundamental vector field $\xi_{\mathcal{V}}$ on $\mathcal{V}$. Let $F(\mathcal{V}) \in \mathcal{A}^{2}(M, \operatorname{End}(\mathcal{V}))$ denote the curvature of $\nabla$. The equivariant curvature is then defined by

$$
F_{\mathfrak{g}}(\mathcal{V}, \xi)=F(\mathcal{V})+2 \pi i \mu(\xi),
$$

and it satisfies the Bianchi identity with respect to the equivariant covariant derivative $\nabla_{\mathfrak{g}}=\nabla+2 \pi i \iota\left(\xi_{M}\right)$. Suppose now that $A \rightarrow f(A)$ is the germ of a $U(N)$-invariant analytic function on $\mathfrak{u}(N)$. Then $f\left(F_{\mathfrak{g}}\right) \in \mathcal{A}_{G}(M)$ is $\mathrm{d}_{\mathfrak{g}}$-closed, and one can show that choosing a different connection changes $f\left(F_{\mathfrak{g}}\right)$ by a $\mathrm{d}_{\mathfrak{g}}$-exact form. The corresponding cohomology classes are called the equivariant characteristic classes of $\mathcal{V} \rightarrow M$. If the action on $M$ is locally free, one can choose $\nabla$ in such a way that $\mu=0$, which shows that the mapping $H_{G}^{\omega}(M) \rightarrow H(M / G)$ sends the equivariant characteristic classes of $\mathcal{V}$ to the usual characteristic classes of the orbifold-bundle $\mathcal{V} / G$.

The following characteristic classes will play a role in the sequel:

(a) The equivariant Chern character, defined by

$$
C h_{\mathfrak{g}}(\mathcal{V}, \xi)=\operatorname{tr}\left(e^{\frac{i}{2 \pi} F_{\mathfrak{g}}(\mathcal{V}, \xi)}\right) .
$$

In the above geometric quantization setting, $\mathcal{V}=L$ is a line bundle, and for the equivariant curvature one has $\frac{i}{2 \pi} F_{\mathfrak{g}}(\mathcal{V}, \xi)=\omega+2 \pi i\langle J, \xi\rangle$, thus

$$
C h_{\mathfrak{g}}(L, \xi)=e^{\omega+2 \pi i\langle J, \xi\rangle} .
$$

More generally, if $g \in G$ acts trivially on the base $M$, one defines

$$
C h_{\mathfrak{g}}^{g}(\mathcal{V}, \xi)=\operatorname{tr}\left(\left(g^{-1}\right)^{\mathcal{V}} e^{\frac{i}{2 \pi} F_{\mathfrak{g}}(\mathcal{V}, \xi)}\right)
$$

where $g^{\mathcal{V}} \in \Gamma(M, \operatorname{End}(\mathcal{V}))$ is the induced action of $g$. In the line bundle case, this is simply $c_{L}\left(g^{-1}\right) C h_{\mathfrak{g}}(L, \xi)$ where $c_{L}(g) \in S^{1}$ is the action of $g$ on the fibers.

(b) The equivariant Todd class,

$$
\operatorname{Td}_{\mathfrak{g}}(\mathcal{V}, \xi)=\operatorname{det}\left(\frac{\frac{i}{2 \pi} F_{\mathfrak{g}}(\mathcal{V}, \xi)}{1-e^{-\frac{i}{2 \pi} F_{\mathfrak{g}}(\mathcal{V}, \xi)}}\right)
$$


The Todd class of a complex manifold is defined as the Todd class of its tangent bundle.

(c) The class

$$
D_{\mathfrak{g}}^{g}(\mathcal{V}, \xi)=\operatorname{det}\left(I-g^{\mathcal{V}} e^{-\frac{i}{2 \pi} F_{\mathfrak{g}}(\mathcal{V}, \xi)}\right),
$$

for $g \in G$ acting trivially on $M$.

All of this also makes sense for symplectic vector bundles, since the choice of a compatible complex structure reduces the structure group to $U(n)$, and any two such choices are homotopic.

We now turn to the equivariant Hirzebruch-Riemann-Roch theorem, in the form due to Berline and Vergne [6]. Let $M$ be a compact complex manifold, equipped with a holomorphic action of $G$, and let $L \rightarrow M$ be a $G$-equivariant holomorphic line bundle. Define the character $\chi \in R(G)$ as in (3).

Theorem 3.1. For $\xi$ sufficiently close to zero,

$$
\chi\left(e^{\xi}\right)=\int_{M} T d_{\mathfrak{g}}(M, \xi) C h_{\mathfrak{g}}(L, \xi) .
$$

More generally, if $g \in G$, one has for all sufficiently small $\xi \in \mathfrak{k}$, the Lie algebra of the centralizer $Z_{g}$ of $g$ :

$$
\chi\left(g e^{\xi}\right)=\int_{M^{g}} \frac{T d_{\mathfrak{k}}\left(M^{g}, \xi\right) C h_{\mathfrak{k}}^{g}\left(L \mid M^{g}, \xi\right)}{D_{\mathfrak{k}}^{g}\left(N^{g}, \xi\right)},
$$

where $M^{g}$ is the fixed point set and $N^{g} \rightarrow M^{g}$ its normal bundle.

To be precise, Berline and Vergne have shown how to rewrite the equivariant Atiyah-Segal-Singer index theorem for Dirac operators in this style, with the equivariant $\hat{A}$-genus appearing on the right hand side. This formula, however, implies the above theorem in the same way as the usual Atiyah-Singer Index theorem leads to the Hirzebruch-Riemann-Roch formula; see [7], p. 152 for the calculations.

Let us now suppose that $(M, \omega)$ is a Hamiltonian $G$-space, such that 0 is a regular value of the moment map $J: M \rightarrow \mathfrak{g}^{*}$. (We do not necessarily assume that $\omega$ is nondegenerate.) Let $M_{0}=J^{-1}(0) / G$ be the symplectic quotient, $\pi: J^{-1}(0) \rightarrow M_{0}$ the projection and $\iota: J^{-1}(0) \rightarrow M$ the embedding. Consider the mapping

$$
\kappa: H_{G}(M) \rightarrow H\left(M_{0}\right),
$$

given by composing pullback to $J^{-1}(0)$ with the mapping $H_{G}^{\omega}\left(J^{-1}(0)\right) \rightarrow H\left(M_{0}\right)$. On the level of forms $\sigma \in \mathcal{A}_{G}^{\omega}(M)$, the form $\pi^{*} \kappa(\sigma)$ is by definition equal to the horizontal part of $\iota^{*} \sigma\left(\frac{i}{2 \pi} \mathrm{d} \theta\right)$, where $\theta \in \mathcal{A}^{1}\left(J^{-1}(0), \mathfrak{g}\right)^{G}$ is some connection.

Let $\mathrm{d} \xi$ be the measure on $\mathfrak{g}$ corresponding to the normalized measure on $G$. The following version of Witten's localization theorem [25] is due to Jeffrey and Kirwan [19] (see also Duistermaat [8] and Vergne [24]).

Theorem 3.2. Let $\sigma \in \mathcal{A}_{G}(M)$ be a $\mathrm{d}_{\mathfrak{g}}$-closed equivariant differential form. Then the integral

$$
f(\xi)=\int_{M} \sigma(\xi) e^{\omega+2 \pi i\langle J, \xi\rangle}
$$

defines a tempered distribution on $\mathfrak{g}$. Its Fourier transform

$$
\hat{f}(\alpha)=\int_{\mathfrak{g}} f(\xi) e^{-2 \pi i\langle\alpha, \xi\rangle} \mathrm{d} \xi
$$


is equal to a polynomial near $\alpha=0$, with value at $\alpha=0$ given by

$$
\hat{f}(0)=\frac{1}{d} \int_{M_{0}} \kappa(\sigma) e^{\omega_{0}} .
$$

where $d$ is the number of elements in the generic stabilizer for the G-action on $J^{-1}(0)$.

Unfortunately, this theorem is only true for polynomial equivariant differential forms, and cannot be applied to the case $\sigma=T d_{\mathfrak{g}}(M) \in \mathcal{A}_{G}^{\omega}(M)$. We will instead use the following stationary phase version. Let $\sigma \in \mathcal{A}_{G}^{\omega}(M)$ be $\mathrm{d}_{\mathfrak{g}}$-closed, and let $\Delta \in C_{0}^{\infty}(\mathfrak{g})$ be a cutoff-function, with $\sigma(\xi)$ defined for $\xi \in \operatorname{supp}(\Delta)$, and $\Delta=1$ in a neighborhood of 0 . Consider the integral

$$
\int_{\mathfrak{g}} \int_{M} \Delta(\xi) \sigma(\xi) e^{m(\omega+2 \pi i\langle J, \xi\rangle)} \mathrm{d} \xi
$$

Since $e^{m \omega}$ is simply a polynomial in $m$, all contributions to the integral which are not $O\left(m^{-\infty}\right)$ come from a neighborhood of the critical set of the phase function $e^{2 \pi i m\langle J, \xi\rangle}$.

Stationarity in the $\mathfrak{g}$-direction gives the condition $J=0$, and stationarity in the $M$-direction the condition $\mathrm{d}\langle J, \xi\rangle=0$, or $\xi=0$ since the action on $J^{-1}(0)$ is locally free.

Theorem 3.3. For $m \rightarrow \infty$,

$$
\int_{\mathfrak{g}} \int_{M} \Delta(\xi) \sigma(\xi) e^{m(\omega+2 \pi i\langle J, \xi\rangle)} \mathrm{d} \xi=\frac{1}{d} \int_{M_{0}} \kappa(\sigma) e^{m \omega_{0}}+O\left(m^{-\infty}\right)
$$

Proof. Since 0 is a regular value of $J$, there exists a $G$-invariant ball $U$ around $0 \in \mathfrak{g}^{*}$ and a $G$-equivariant diffeomorphism $\phi: J^{-1}(0) \times U \rightarrow J^{-1}(U)$, such that $\phi^{*} J=\operatorname{pr}_{2}$ is projection onto the second factor. Let $\chi \in C_{0}^{\infty}(U)$ be equal to 1 near 0 . Using the mapping $\phi$ and the stationary phase theorem, the above integral is equal to

$$
\int_{\mathfrak{g}} \int_{X} \Delta(\xi) \chi(\alpha) \phi^{*} \sigma(\xi) e^{m\left(\phi^{*} \omega+2 \pi i\langle\alpha, \xi\rangle\right)} \mathrm{d} \xi+O\left(m^{-\infty}\right),
$$

where $X=J^{-1}(0) \times U$. Since $X$ retracts to $J^{-1}(0)$, there exists a form $\tau \in \mathcal{A}_{G}^{\omega}(X)$ such that $\phi^{*} \sigma-\operatorname{pr}_{1}^{*} \iota^{*} \sigma=\mathrm{d}_{\mathfrak{g}} \tau$. By Stokes' theorem,

$$
\int_{X} \chi \mathrm{d}_{\mathfrak{g}} \tau(\xi) e^{m(\omega+2 \pi i\langle J, \xi\rangle)}=-\int_{X}\left(\mathrm{~d}_{\mathfrak{g}} \chi\right) \tau(\xi) e^{m(\omega+2 \pi i\langle J, \xi\rangle)}=O\left(m^{-\infty}\right)
$$

because $\mathrm{d}_{\mathfrak{g}} \chi=0$ near 0 , so we can replace $\phi^{*} \sigma$ by $\operatorname{pr}_{2}^{*} \iota^{*} \sigma$. Similarly, there exists a $G$-invariant form $\beta \in \mathcal{A}^{1}(X)$ such that $\phi^{*} \omega-\operatorname{pr}_{1}^{*} \pi^{*} \omega_{0}=\mathrm{d} \beta$, and such that the pullback of $\beta$ to $J^{-1}(0)$ is zero. Since

$$
-\mathrm{d}\left\langle\phi^{*} J, \xi\right\rangle=\phi^{*} \iota\left(\xi_{M}\right) \omega=\iota\left(\xi_{X}\right) \phi^{*} \omega=\iota\left(\xi_{X}\right) \mathrm{d} \beta=\mathrm{d} \iota\left(\xi_{X}\right) \beta,
$$

we know that $\iota\left(\xi_{X}\right) \beta=-\langle\alpha, \xi\rangle$, and therefore $\rho:=\beta+\langle\alpha, \theta\rangle$ is basic on $X$. In particular, $\mathrm{d}_{\mathfrak{g}} \rho=\mathrm{d} \rho$. Since $e^{m d \rho}-1$ is a $\mathrm{d}_{\mathfrak{g}}$-exact polynomial in $m$, we may replace $e^{m \phi^{*} \omega}$ by $e^{m\left(\pi^{*} \omega_{0}+\mathrm{d}\langle\alpha, \theta\rangle\right)}$ in the integral. We hence find that the integral is equal to

$$
m^{\operatorname{dim} G} \int_{J^{-1}(0)} \int_{\mathfrak{g}^{*}} \int_{\mathfrak{g}} \Delta(\xi) \chi(\alpha)\left(\iota^{*} \sigma\right)(\xi) e^{m\left(\pi^{*} \omega_{0}+\langle\alpha, \mathrm{d} \theta+2 \pi i \xi\rangle\right)} \mathrm{d} \xi \mathrm{d} \alpha \mathrm{d} g+O\left(m^{-\infty}\right) .
$$


Here, $\mathrm{d} g$ denotes the (vertical) volume form on the fibers of $J^{-1}(0) \rightarrow M_{0}$, corresponding to the canonical identification $T_{x}$ (fiber) $\cong \mathfrak{g}$ by means of the $G$-action. Now apply the stationary phase theorem (see e.g. [18], Theorem 7.7.5) to the $\alpha, \xi$-integral, the relevant phase function being $e^{2 \pi i m\langle\alpha, \xi\rangle}$. Since $e^{m\langle\alpha, \mathrm{d} \theta\rangle}$ is simply a polynomial in $\alpha$, the stationary phase expansion terminates after finitely many terms, and the result is

$$
\begin{gathered}
\left.\int_{J^{-1}(0)} e^{m \pi^{*} \omega_{0}} \sum_{r=0}^{\infty} \frac{1}{r !}\left(\frac{i}{2 \pi m}\right)^{r}\left(\sum_{j} \frac{\partial}{\partial \xi_{j}} \frac{\partial}{\partial \alpha^{j}}\right)^{r}\right|_{\substack{\xi=0 \\
\alpha=0}} \iota^{*} \sigma(\xi) e^{m\langle\alpha, \mathrm{d} \theta\rangle} \mathrm{d} g+O\left(m^{-\infty}\right) \\
=\int_{J^{-1}(0)} \pi^{*}\left(e^{m \omega_{0}}\right) \iota^{*} \sigma\left(\frac{i}{2 \pi} \mathrm{d} \theta\right) \mathrm{d} g+O\left(m^{-\infty}\right) .
\end{gathered}
$$

Since $\iota^{*} \sigma\left(\frac{i}{2 \pi} \mathrm{d} \theta\right)$ gets wedged with $\mathrm{d} g$, only its horizontal part, which by definition of $\kappa$ is $\pi^{*} \kappa(\sigma)$, contributes to the integral. The result (38) now follows by integration over the fiber; the factor $1 / d$ appears since this is the volume of a generic fiber.

\section{The STATIONARY PHASE APPROXIMATION}

In this section, we will prove the first part of Theorem 2.1. By the shifting-trick, it is sufficient to consider the case $\mu=0$. The idea is to substitute the expressions from the equivariant Hirzebruch-Riemann-Roch Theorem 3.1 for $\chi^{(m)}$ in

$$
N^{(m)}(0)=\int_{G} \chi^{(m)}(h) \mathrm{d} h,
$$

and apply the localization formula, Theorem 3.3. For this, we need to know what happens to the equivariant Todd class of $M$ under the mapping (34):

Lemma 4.1. Let

$$
j_{\mathfrak{g}}(\xi)=\operatorname{det}\left(\frac{1-e^{-\operatorname{ad}(\xi)}}{\operatorname{ad}(\xi)}\right)
$$

be the Jacobian of the exponential mapping $\exp : \mathfrak{g} \rightarrow G$. Then

$$
\kappa\left(T d_{\mathfrak{g}}(M) j_{\mathfrak{g}}\right)=\operatorname{Td}\left(M_{0}\right) .
$$

Proof. Identify the vertical subbundle of $T J^{-1}(0)$ with the trivial bundle $\mathfrak{g}$, and the symplectic bundle $\mathfrak{g} \oplus I \mathfrak{g}$ (where $I$ is the complex structure of $M$ ) with $\mathfrak{g}_{\mathbb{C}}$. Then

$$
\iota^{*}(T M)=\pi^{*}\left(T M_{0}\right) \oplus \mathfrak{g}_{\mathbb{C}} .
$$

Since the equivariant Todd class of $\mathfrak{g}_{\mathbb{C}}$ is just $j_{\mathfrak{g}}^{-1}$, this shows that $\kappa\left(T d_{\mathfrak{g}}(M)\right)=$ $\operatorname{Td}\left(M_{0}\right) \kappa\left(j_{\mathfrak{g}}^{-1}\right)$, q.e.d.

We will now consider the contribution to (39) coming from a small Ad-invariant neighborhood of a given orbit $(g)=\operatorname{Ad}(G) . g$. Let $\sigma \in C^{\infty}(G)$ be an Ad-invariant cutoff-function, supported in a sufficiently small neighborhood of $(g)$ and equal to 1 near $(g)$. Consider the integral

$$
I_{g}(m)=\int_{G} \sigma(h) \chi^{(m)}(h) \mathrm{d} h .
$$

Since (33) only holds for $\xi \in \mathfrak{k}$, we want to replace this integral by an integral over $Z_{g}$. (Of course, this step is void in the abelian case.) 
Let $\mathfrak{r} \subset \mathfrak{g}$ be the orthogonal complement of $\mathfrak{k}$ with respect to some invariant inner product (or, more intrinsically, the annihilator of $\left(\mathfrak{g}^{*}\right)^{g} \cong \mathfrak{k}^{*}$ ). For $k \in Z_{g}$, let $k^{\mathfrak{r}}$ denote the action of $k$ on $\mathfrak{r}$.

Lemma 4.2. Let $f \in C^{\infty}(G)$ be $\operatorname{Ad}(G)$-invariant, with support in a small neighborhood of $\operatorname{Ad}(G) . g$. Then, for a suitable $\operatorname{Ad}\left(Z_{g}\right)$-invariant cutoff-function $\tilde{\sigma} \in$ $C_{0}^{\infty}\left(Z_{g}\right)$, supported near $e \in Z_{g}$ and identically 1 near $e$,

$$
\int_{G} f(h) \mathrm{d} h=\int_{Z_{g}} f(g k) \operatorname{det}\left(I-(g k)^{\mathfrak{r}}\right) \tilde{\sigma}(k) \mathrm{d} k .
$$

(For a proof, see e.g. [10].) Using the lemma, we find that

$$
I_{g}(m)=\int_{Z_{g}} \tilde{\sigma}(k) \chi^{(m)}(g k) \operatorname{det}\left(I-(g k)^{\mathfrak{r}}\right) \mathrm{d} k .
$$

Replacing this with an integral over the Lie algebra $\mathfrak{k}$, and using (33) gives

$$
I_{g}(m)=\int_{\mathfrak{k}} \int_{M^{g}} \Delta(\xi) \frac{T d_{\mathfrak{k}}\left(M^{g}, \xi\right) C h_{\mathfrak{k}}^{g}\left(L^{m} \mid M^{g}, \xi\right)}{D_{\mathfrak{k}}^{g}\left(N^{g}, \xi\right)} \operatorname{det}\left(I-\left(g e^{\xi}\right)^{\mathfrak{r}}\right) j_{\mathfrak{k}}(\xi) \mathrm{d} \xi,
$$

with $\Delta(\xi)=\tilde{\sigma}\left(e^{\xi}\right)$. Let $\kappa_{g}: H_{Z_{g}}\left(M^{g}\right) \rightarrow H\left(\Sigma_{g}\right)$ be the mapping defined by (34), with $M$ replaced by $M^{g}$ and $G$ by $Z_{g}$. By Lemma 4.1,

$$
\kappa_{g}\left(C h_{\mathfrak{k}}^{g}\left(L^{m} \mid M^{g}\right)\right) \kappa_{g}\left(j_{\mathfrak{k}}\right)=C h^{\Sigma}\left(L_{\Sigma}^{m}\right) \mid \Sigma_{g} .
$$

For $x \in P^{g}=M^{g} \cap J^{-1}(0)$, let $\mathfrak{r}_{M}(x):=\left\{\xi_{M}(x) \mid \xi \in \mathfrak{r}\right\} \cong \mathfrak{r}$. Then

$$
N^{g}(x)=N_{\Sigma}(y) \oplus \mathfrak{r}_{M}(x) \oplus I \mathfrak{r}_{M}(x)=N_{\Sigma}(y) \oplus \mathfrak{r}_{\mathbb{C}},
$$

where $y=G .(x, g)$. But $D_{\mathfrak{k}}^{g}\left(\mathfrak{r}_{\mathbb{C}}, \xi\right)=\operatorname{det}\left(I-\left(g e^{\xi}\right)\right)$, hence

$$
\kappa_{g}\left(D_{\mathfrak{k}}^{g}\left(N^{g}, \xi\right)\right)=\kappa_{g}\left(\operatorname{det}\left(I-\left(g e^{\xi}\right)^{\mathfrak{r}}\right)\right) D^{\Sigma}\left(N_{\Sigma}\right) \mid \Sigma_{g} .
$$

With these preparations, we apply Theorem 3.3 to the integral (45), and obtain

$$
I_{g}(m)=\sum_{j}^{\prime} \frac{1}{d_{j}} \int_{\Sigma_{j}} \frac{T d\left(\Sigma_{j}\right) C h^{\Sigma}\left(L_{\Sigma}^{m}\right)}{D^{\Sigma}\left(N_{\Sigma}\right)}+O\left(m^{-\infty}\right),
$$

the sum being over the connected components of $\Sigma_{g}$. Summing over all contributions, we get (15) up to an error term $O\left(m^{-\infty}\right)$. As we remarked above, the right hand side of (15) is an arithmetic polynomial as a function of $m$. But if $f: \mathbb{N} \rightarrow \mathbb{Z}$ is an integer-valued function with $\lim _{m \rightarrow \infty}(f(m)-p(m))=0$ for some polynomial $p$, then $f(m)=p(m)$ for large $m$. This shows that the error term is zero for large $m$, and finishes the proof of the first part of Theorem 2.1.

\section{Counting lattice Points}

To prove the second part of Theorem 2.1, i.e. that we can set $m=1$, all we have to show is that $m \rightarrow N^{(m)}(m \mu)$ is an arithmetic polynomial.

Theorem 5.1. Suppose that $J(M) \subset \mathfrak{g}_{\text {reg. }}^{*}$. Then the function $m \mapsto N^{(m)}(m \mu)$ is an arithmetic polynomial for all $\mu \in \Lambda_{+}^{*}$.

Before we prove this, we convert the computation of the multiplicities into a problem of counting lattice points. The next steps are based on work of GuilleminLerman-Sternberg [14] and Guillemin-Prato [15], except that we replace their use of the Atiyah-Bott Lefschetz formula with the equivariant index theorem, since we do not want to assume isolated fixed points. Consider the action of the maximal 
torus $T \subset G$, with its moment map $J^{T}$ equal to $J$ followed by projection to $\mathfrak{t}^{*}$. The following is a special case of (5):

Proposition 5.1. Let $\xi \in \mathfrak{t}$ be generic in the sense that the zero set of $\xi_{M}$ is equal to the fixed point set of the T-action. Then

$$
\chi\left(e^{\xi}\right)=\sum_{\mathcal{F}} \int_{\mathcal{F}} \frac{T d(\mathcal{F}) e^{\omega+2 \pi i\left\langle J_{\mathcal{F}}, \xi\right\rangle}}{\operatorname{det}\left(I-e^{-\frac{i}{2 \pi} F_{\mathfrak{t}}\left(N_{\mathcal{F}}, \xi\right)}\right)},
$$

the sum being over the fixed points manifolds of the $T \subset G$-action, $N_{\mathcal{F}}$ the corresponding normal bundles, and $J_{\mathcal{F}}$ the constant value of $J$ on $\mathcal{F}$.

Although the left hand side of (50) is an analytic function of $\xi$, the individual summands on the right hand side have poles. Since they are not in $L_{l o c}^{1}$, they do not a priori define distributions on $\mathfrak{t}$. This problem can be fixed as follows [8]. By using the splitting principle (or simply a partition of unity on $\mathcal{F}$ ) if necessary, we can assume that $N_{\mathcal{F}}$ splits into a direct sum of invariant line bundles $N_{\mathcal{F}}^{1}, \ldots, N_{\mathcal{F}}^{r}$. Let $\alpha_{\mathcal{F}}^{j}$ be the weight for the $T$-action on $N_{\mathcal{F}}^{j}$, that is, $e^{\xi} \in T$ acts by the character $\exp \left(2 \pi i\left\langle\alpha_{\mathcal{F}}^{j}, \xi\right\rangle\right)$. Each $\alpha_{\mathcal{F}}^{j}$ determines an orthogonal hyperplane in $\mathfrak{t}$; let $C$ be any fixed connected component in the complement of the union of all these hyperplanes. If we replace $\xi$ by $\xi-i \eta$ in (50), with $\eta \in C$, the terms on the right hand side are analytic for all $\xi$. It is therefore possible to regard (50) as an equality of distributions, with the summands on the right hand side defined as a limit for $\eta \rightarrow 0$ in $C$.

Let us now first discuss the abelian case, i.e. assume that $G=T$ is a torus. Denote by $F\left(N_{\mathcal{F}}^{j}\right)$ the components of the curvature. By expanding

$$
\operatorname{det}\left(I-e^{-\frac{i}{2 \pi} F_{\mathfrak{t}}\left(N_{\mathcal{F}}, \xi-i \eta\right)}\right)^{-1}
$$

into its Taylor series with respect to $F\left(N_{\mathcal{F}}^{j}\right)$, we can write it as a finite sum

$$
\operatorname{det}\left(I-e^{-\frac{i}{2 \pi} F_{\mathbf{t}}(N, \xi-i \eta)}\right)^{-1}=\sum_{s \in \mathbb{N}^{r}} \frac{p_{s}\left(F\left(N_{\mathcal{F}}^{1}\right), \ldots, F\left(N_{\mathcal{F}}^{r}\right)\right)}{\prod_{j}\left(1-e^{-2 \pi i\left\langle\alpha_{\mathcal{F}}^{j}, \xi-i \eta\right\rangle}\right)^{s_{j}}},
$$

where for all $s=\left(s_{1}, \ldots, s_{r}\right), p_{s}$ is a polynomial. We now invoke the "polarization trick" used in $[14,15]$. For each $\alpha_{\mathcal{F}}^{j}$, write

$$
\check{\alpha}_{\mathcal{F}}^{j}= \begin{cases}\alpha_{\mathcal{F}}^{j} & \text { if }\left\langle\alpha_{\mathcal{F}}^{j}, \eta\right\rangle>0 \\ -\alpha_{\mathcal{F}}^{j} & \text { if }\left\langle\alpha_{\mathcal{F}}^{j}, \eta\right\rangle<0\end{cases}
$$

for any, hence all, $\eta \in C$. Let $l_{j}^{0}=0$ if $\check{\alpha}_{\mathcal{F}}^{j}=\alpha_{\mathcal{F}}^{j}, 1$ otherwise. Then

$$
\chi\left(e^{\xi-i \eta}\right)=\sum_{\mathcal{F}} \sum_{s \in \mathbb{N}^{r}} c_{\mathcal{F}, s} \frac{e^{2 \pi i\left\langle J_{\mathcal{F}}-\sum l_{j}^{0} s_{j} \check{\alpha}_{\mathcal{F}}^{j}, \xi-i \eta\right\rangle}}{\prod_{j}\left(1-e^{-2 \pi i\left\langle\check{\alpha}_{\mathcal{F}}^{j}, \xi-i \eta\right\rangle}\right)^{s_{j}}}
$$

with

$$
c_{\mathcal{F}, s}=(-1)^{k_{\mathcal{F}, s}} \int_{\mathcal{F}} \operatorname{Td}(\mathcal{F}) e^{\omega} p_{s}\left(F^{1}\left(N_{\mathcal{F}}\right), \ldots, F^{r}\left(N_{\mathcal{F}}\right)\right),
$$

where $k_{\mathcal{F}, s}=\sum l_{j}^{0} s_{j}$ is the number of sign changes. 
For given $\mathcal{F}, s$, write $\left(a^{1}, \ldots, a^{N}\right)$ for the list of $\check{\alpha}_{\mathcal{F}}^{j}$ 's, appearing with respective multiplicities $s_{j}$. Since

$$
\left(1-e^{-2 \pi i\left\langle a^{j}, \xi-i \eta\right\rangle}\right)^{-1}=\sum_{l_{j}=0}^{\infty} e^{-2 \pi i\left\langle l_{j} a^{j}, \xi-i \eta\right\rangle},
$$

we get

$$
\chi\left(e^{\xi}\right)=\sum_{\mathcal{F}, s} c_{\mathcal{F}, s} \sum_{l \in \mathbb{Z}_{+}^{N}} e^{2 \pi i\left\langle J_{\mathcal{F}}-\sum\left(l_{j}+l_{j}^{0}\right) a^{j}, \xi\right\rangle}
$$

(the sum over $\mathbb{Z}_{+}^{N}:=\left\{l \in \mathbb{Z}^{N}: l_{j} \geq 0\right\}$ is a well-defined periodic distribution). Comparing this to

$$
\chi\left(e^{\xi}\right)=\sum_{\mu \in \Lambda} N(\mu) e^{2 \pi i\langle\mu, \xi\rangle}
$$

yields

$$
N(\mu)=\sum_{\mathcal{F}, s} c_{\mathcal{F}, s} \mathfrak{P}_{\mathcal{F}, s}\left(J_{\mathcal{F}}-\mu-\sum l_{j}^{0} a^{j}\right)
$$

where the partition function $\mathfrak{P}_{\mathcal{F}, s}(\nu)$ is the number of solutions $k \in \mathbb{Z}^{N}$ of $\sum k_{j} a^{j}=$ $\nu, k_{j} \geq 0$.

Starting from this expression, we will now show that $N^{(m)}(m \mu)$ is an arithmetic polynomial. We have to replace $\omega$ by $m \omega, \mu$ by $m \mu$ and $J$ by $m J$. Since $c_{\mathcal{F}, s}^{(m)}$ is a polynomial in $m$, it is sufficient to show that the number of integer solutions of

$$
m\left(J_{\mathcal{F}}-\mu\right)=\sum\left(l_{j}+l_{j}^{0}\right) a^{j}, l_{j} \geq 0
$$

is an arithmetic polynomial as a function of $m$. Let us write $\nu=J_{\mathcal{F}}-\mu$, and consider $A=\left(a^{1}, \ldots, a^{N}\right)$ as a $\mathbb{Z}$-linear mapping $\mathbb{Z}^{N} \rightarrow \mathbb{Z}^{p}$, where $p=\operatorname{dim}(T)$. We are thus looking for integer solutions of

$$
m \nu=A l, l_{j} \geq l_{j}^{0} .
$$

We will need the following

Theorem 5.2 (Ehrhart [11]). Let $L$ be a lattice, with underlying vector space $L_{\mathbb{R}}=$ $L \otimes_{\mathbb{Z}} \mathbb{R}$, and $\Delta \subset L_{\mathbb{R}}$ a lattice polytope, i.e. a polytope whose vertices are all lattice points. Then, for all $r \in \mathbb{N}$, the counting function

$$
f(m)=\#\left(\frac{m}{r} \Delta \cap L\right)
$$

is an arithmetic polynomial, with period $r$.

Let now $x_{0} \in \mathbb{R}^{N}$ be any solution of $A x=\nu$. The general solution of $A x=m \nu$ is thus given by the affine plane $E_{m}=m x_{0}+\operatorname{ker}(A)$. Let $r \in \mathbb{N}$ be the smallest number such that the vertices of the polytope $\Delta:=E_{r} \cap \mathbb{R}_{+}^{N}$ are lattice points. If $l^{0}=0$, the set of solutions of (60) is the intersection $\frac{m}{r} \Delta \cap \mathbb{Z}^{N}$, so the number of solutions is an arithmetic polynomial by Ehrhart's theorem. If $l^{0} \neq 0$, let $\Delta_{j}$ be the face of $\Delta$ defined by $x_{j}=0$, and let $\Delta^{\prime}$ be the union of all $\Delta_{j}$ for which $l_{j}^{0}=1$. Then the solution set of $(60)$ is

$$
\frac{m}{r} \Delta \cap \mathbb{Z}^{N}-\frac{m}{r} \Delta^{\prime} \cap \mathbb{Z}^{N}
$$

and this gives again an arithmetic polynomial by Ehrhart's theorem. This proves Theorem 5.1 in the abelian case. 
Suppose now that $G$ is nonabelian, but that $J(M)$ is contained in the set of regular elements, $\mathfrak{g}_{\text {reg }}^{*}=G$.int $\left(\mathfrak{t}_{+}^{*}\right)$. We will show how this reduces to the abelian case. By the symplectic slice theorem [17], $Y_{+}=J^{-1}\left(\operatorname{int}\left(\mathfrak{t}_{+}^{*}\right)\right)$ is a symplectic (but not necessarily Kähler) submanifold of $M$, and is in fact a Hamiltonian $T$-space, with the restriction of $J$ serving as a moment map. The above assumption implies that $Y_{+}$is a closed submanifold, and $M=G \times_{T} Y_{+}$. The restriction $L_{+}=L \mid Y_{+}$ yields a quantizing bundle for $Y_{+}$. Consider the expression

$$
\chi^{\prime}\left(e^{\xi}\right):=\int_{Y^{+}} T d_{\mathfrak{t}}\left(Y_{+}, \xi\right) C h_{\mathfrak{t}}\left(L_{+}, \xi\right) .
$$

We claim that this is of the form

$$
\chi^{\prime}\left(e^{\xi}\right)=\sum_{\mu \in \Lambda} N^{\prime}(\mu) e^{2 \pi i\langle\mu, \xi\rangle},
$$

where $N^{\prime}(\mu) \neq 0$ for only finitely many lattice points, and $N^{\prime}(\mu)=0$ unless $\mu \in$ $J\left(Y_{+}\right) \cap \Lambda \subset \Lambda_{+}^{*}$. Indeed, one can check directly that $\chi\left(e^{\xi}\right)$ comes from a function on $T$, given near any point $g \in T$ by the formula (33), and then repeat the above analysis. (One can also pick a $T$-invariant almost Kähler structure on $M$, and then realize $\chi\left(e^{\xi}\right)$ as the equivariant index of the $\operatorname{Spin}^{c}$-Dirac operator associated to $L \mid Y_{+} \rightarrow Y_{+}$.)

Lemma 5.1. For all $\mu \in \Lambda_{+}^{*}, N(\mu)=N^{\prime}(\mu)$.

Since we know that $N^{\prime(m)}(m \mu)$ is an arithmetic polynomial, this will finish the proof of Theorem 2.1 .

Proof. Let us go back to the formula (50) for the character. Notice that the Weyl group $W=N_{G}(T) / T$ acts on $M^{T}$ by permuting the connected components, and that $M^{T}$ consists of its portion in $Y_{+}$and the $W$-transforms thereof. Let $\mathcal{F} \subset Y_{+}$ be a connected component of $M^{T}$. The normal bundle $N_{\mathcal{F}}$ of $\mathcal{F}$ in $M$ splits into its part in $Y_{+}, N_{\mathcal{F}}^{\prime}:=N_{\mathcal{F}} \cap T Y_{+}$, and the symplectic orthogonal complement of $T Y_{+} \mid \mathcal{F}$, which is canonically isomorphic to the trivial bundle $\mathfrak{g} / \mathfrak{t}$. The weights for the $T$-action on $\mathfrak{g} / \mathfrak{t}$ are of course simply the positive roots $\beta \in \mathfrak{t}^{*}$ of $G$. Therefore, by taking the trivial connection on $\mathfrak{g} / \mathfrak{t}$,

$$
\operatorname{det}\left(I-e^{-\frac{i}{2 \pi} F_{\mathfrak{t}}\left(N_{\mathcal{F}}, \xi\right)}\right)=\prod_{\beta>0}\left(1-e^{-2 \pi i\langle\beta, \xi\rangle}\right) \operatorname{det}\left(I-e^{-\frac{i}{2 \pi} F_{\mathbf{t}}\left(N_{\mathcal{F}}^{\prime}, \xi\right)}\right),
$$

hence

$$
\chi\left(e^{\xi}\right)=\sum_{w \in W} \frac{1}{\prod_{\beta>0}\left(1-e^{-2 \pi i\left\langle\beta, w^{-1}(\xi)\right\rangle}\right)} \sum_{\mathcal{F} \subset Y_{+}} \int_{\mathcal{F}} \frac{T d(\mathcal{F}) e^{\omega+2 \pi i\left\langle J_{\mathcal{F}}, w^{-1}(\xi)\right\rangle}}{\operatorname{det}\left(I-e^{-\frac{i}{2 \pi} F_{\mathfrak{t}}\left(N_{\mathcal{F}}^{\prime}, w^{-1}(\xi)\right)}\right)} .
$$

We can apply the localization formula to the sum

$$
\sum_{\mathcal{F} \subset Y_{+}} \int_{\mathcal{F}} \frac{T d(\mathcal{F}) e^{\omega+2 \pi i\left\langle J_{\mathcal{F}}, \xi\right\rangle}}{\operatorname{det}\left(I-e^{-\frac{i}{2 \pi} F_{\mathrm{t}}\left(N_{\mathcal{F}}^{\prime}, \xi\right)}\right)}
$$

this time in the opposite direction, and find that it is equal to the above expression $\chi^{\prime}\left(e^{\xi}\right)$.

Hence

$$
\chi\left(e^{\xi}\right)=\sum_{w \in W} \frac{\chi^{\prime}\left(e^{w^{-1}(\xi)}\right)}{\prod_{\beta>0}\left(1-e^{-2 \pi i\left\langle\beta, w^{-1}(\xi)\right\rangle}\right)} .
$$


But

$$
\prod_{\beta>0}\left(1-e^{-2 \pi i\left\langle\beta, w^{-1}(\xi)\right\rangle}\right)=\operatorname{det}(w) e^{-2 \pi i\langle w(\delta)-\delta, \xi\rangle} \prod_{\beta>0}\left(1-e^{-2 \pi i\langle\beta, \xi\rangle}\right),
$$

where $\delta=\frac{1}{2} \sum_{\beta>0} \beta$ is the magic weight. Weyl's character formula hence shows that

$$
\chi\left(e^{\xi}\right)=\sum_{\mu \in \Lambda_{+}^{*}} N^{\prime}(\mu) \sum_{w \in W} \operatorname{det}(w) \frac{e^{2 \pi i\langle w(\delta+\mu)-\delta, \xi\rangle}}{\prod_{\beta>0}\left(1-e^{-2 \pi i\langle\beta, \xi\rangle}\right)}=\sum_{\mu \in \Lambda_{+}^{*}} N^{\prime}(\mu) \chi_{\mu}\left(e^{\xi}\right),
$$

where $\chi_{\mu}$ is the character of the irreducible representation corresponding to $\mu$. This proves $N(\mu)=N^{\prime}(\mu)$.

Remarks. (1) If $J(M) \not \subset \mathfrak{g}_{\text {reg }}^{*}$, it is still possible to derive a formula for $N(\mu)$ similar to (58), following part II of Guillemin-Prato [15]. However, this formula involves an additional "shift", so that (60) gets replaced by an equation of the form

$$
A l=m \nu+\sigma, l_{j} \geq l_{j}^{0} .
$$

In general, the number of integer solutions of such an equation is not an arithmetic polynomial for all $m \in \mathbb{N}$, even though this is true for large $m$.

(2) On the other hand, Theorem 5.1 does not require that $\mu$ is a regular value of $J$. Even in the singular case, it is therefore sufficient to prove multiplicity formulas under the assumption $m>>0$.

\section{REFERENCES}

1. M. F. Atiyah: Elliptic operators and compact groups, Lecture Notes in Mathematics 401, Springer-Verlag (1974). MR 58:2910

2. M. F. Atiyah, R. Bott: The moment map and equivariant cohomology, Topology 23, 1-28 (1984). MR 85e:58041

3. M. F. Atiyah, G. Segal: The index of elliptic operators II. Ann. Math. 87, 531-545 (1968). MR 38:5244

4. M. F. Atiyah, I. Singer: The index of elliptic operators III. Ann. Math. 87, 546-604 (1968). MR 38:5245

5. N. Berline, M. Vergne: Classes charactéristiques équivariantes. Formules de localisation en cohomologie équivariante, C. R. Acad. Sci. Paris 295, 539-541 (1982). MR 83m:58002

6. N. Berline, M. Vergne: The equivariant index and Kirillov's character formula, Am. J. Math. 107, 1159-1190 (1985). MR 87a:58143

7. N. Berline. E. Getzler, M. Vergne: Heat kernels and Dirac operators, Springer-Verlag 1992. MR 94e:58130

8. J. J. Duistermaat: Equivariant cohomology and stationary phase, Symplectic Geometry and Quantization (Sanda and Yokohama, 1993), Contemp. Math., vol. 179, Amer. Math. Soc., Providence, RI, 1994, pp. 45-62. CMP 95:09

9. J. J. Duistermaat, G. Heckman: On the variation in the cohomology of the symplectic form of the reduced phase space, Inv. Math. 69, 259-268 (1982). Addendum, 72, 153-158 (1983). MR 84h:58051a,b

10. M. Duflo, M. Vergne: Cohomologie équivariante et descente, Asterisque 215 (1993). MR 95f: 22018

11. E. Ehrhart: Polynômes arithméthiques et méthode des polyèdres en combinatoire, Birkhäuser Verlag, 1977. MR 55:5544

12. C. Farsi: K-theoretical Index theorems for orbifolds, Quart. J. Math. Oxford 43 (2), 183-200 (1992). MR 93f:58231

13. V. Guillemin: Reduction and Riemann-Roch, In: Lie groups and geometry in honour of B. Kostant. Progr. Math. Birkhäuser Boston, 1994. 
14. V. Guillemin, E. Lerman, S. Sternberg: On the Kostant multiplicity formula J. Geom. Phys. 5, 721-750 (1988). MR 92f:58058

15. V. Guillemin, E. Prato: Heckman, Kostant and Steinberg formulas for symplectic manifolds, Adv. Math. 82, 160-179 (1990). MR 91h:58041

16. V. Guillemin, S. Sternberg: Geometric quantization and multiplicities of group representations, Invent. Math. 67, 515-538 (1982). MR 83m:58040

17. V. Guillemin, S. Sternberg:Symplectic techniques in physics, Cambridge University Press 1984. MR 86f:58054

18. L. Hörmander: The analysis of linear partial differential operators I. Springer 1990. MR 91m:35001a

19. L. Jeffrey, F. Kirwan: Localization for nonabelian group actions, Topology 34, 291-327 (1995). CMP 95:08

20. T. Kawasaki: The Riemann-Roch theorem for complex V-manifolds, Osaka J. Math. 16 151-157 (1979). MR 80f:58042

21. I. Satake: The Gauss-Bonnet theorem for V-manifolds, J. Math. Soc. Japan, 9, 464-492 (1957). MR 20:2022

22. R. Sjamaar: Holomorphic slices, symplectic reduction and multiplicities of representations, Ann. Math. 141, 87-129 (1995). CMP 95:07

23. M. Vergne: Quantification géométrique et multiplicités, C. R. Acad. Sci., 319, 327-332 (1994). CMP 94:16

24. M. Vergne: A note on Jeffrey-Kirwan-Witten's localization formula, Preprint DMI, École Norm. Sup., Paris (1994).

25. E. Witten: Two dimensional gauge theories revisited, J. Geom. Phys. 9 (1992), 303-368. MR 93m:58017

\begin{abstract}
A theorem of Guillemin and Sternberg about geometric quantization of Hamiltonian actions of compact Lie groups $G$ on compact Kähler manifolds says that the dimension of the $G$-invariant subspace is equal to the Riemann-Roch number of the symplectic quotient. Combined with the shifting-trick, this gives explicit formulas for the multiplicities of the various irreducible components. One of the assumptions of the theorem is that the reduction is regular, so that the reduced space is a smooth symplectic manifold. In this paper, we prove a generalization of this result to the case where the reduced space may have orbifold singularities. The result extends to nonKählerian settings, if one defines the representation by the equivariant index of the Spin ${ }^{c}$-Dirac operator associated to the quantizing line bundle.
\end{abstract}

Department of Mathematics, Massachusetts institute of Technology, Cambridge, MASSACHUSETTS 02139 